\title{
25 AÑOS DESPUÉS DEL ESTRENO DE PHILADELPHIA (1993), ¿QUÉ COSAS HAN CAMBIADO EN LA INFECCIÓN POR VIH?
}

\section{5 years after Philadelphia (1993) premiere, what things have changed in HIV infection?}

\section{Emilio PINTOR HOLGUÍN; Benjamín HERREROS RUIZ-VALDEPEÑAS; Pedro GARGANTILLA MADERA; María Josefa GUTIÉRREZ CISNEROS}

Universidad Europea de Madrid. Facultad de Ciencias Biomédicas. Villaviciosa de Odón (España).

e-mail: emilio.pintor@universidadeuropea.es

Fecha de recepción: 25 de junio de 2019

Fecha de aceptación: 5 de septiembre de 2019

Fecha de publicación: 15 de marzo de 2020

\section{Resumen}

En el presente año 2019 se han cumplido 25 años del estreno de la película Philadelhia de Jonathan Demme, que en nuestro país se produjo en marzo de 1994. Pretendemos con este artículo hacer una reflexión de los cambios que se han producido en la infección HIV tanto en el diagnóstico, tratamiento y pronostico así como en el ámbito social: cuando se presenta la película hacía unos 10 años de los primeros casos, se contemplaba la infección por HIV como una enfermedad con un amplio rechazo social con estigmatización de varios grupos de riesgo (homosexuales, adictos por vía intravenosa y hemofílicos) y las vías de contagio, el diagnóstico solía hacerse en fases tardías de la enfermedad con cuadros de inmunodeficiencia severa como sarcoma de Kaposi, neumonía por Pneumocystis carinii, toxoplasmosis cerebral y meningitis por criptococo y los tratamientos eran poco efectivos. En la actualidad, el diagnóstico y tratamiento es mucho más precoz y efectivo y la estigmatización social ha desaparecido.

Palabras clave: infección por HIV; SIDA; tratamiento; infecciones oportunistas; estigmatización social.

\section{Summary}

In present year 2019, 25 years of the premiere of the film Philadelhia by Jonathan Demme have been fulfilled, which in our country took place in March 1994. With this paper, we try to make a reflection of the changes that have occurred in HIV infection in diagnosis, treatment and prognosis as well as in the social field: when the film was presented about 10 years later first patients were diagnosed, HIV infection was considered as a disease with wide-spread social rejection with stigmatization of several risk groups (homosexuals, intravenous drug users and hemophiliacs) and ways of transmission, diagnosis was usually made in late stages of the disease with severe 
immunodeficiency symptoms such as Kaposi's sarcoma, Pneumocystis carinii pneumonia, cerebral toxoplasmosis and cryptococcal meningitis and the treatments were ineffective. At present, diagnosis and treatment is much earlier and more effective and social stigmatization has disappeared.

Key words: HIV infection; AIDS; treatment; opportunistic infections; social stigmatization.

\section{Ficha técnica}

Título original: Philadelphia.

País: Estados Unidos.

Año: 1993.

Director: Jonathan Demme.

Música: Howard Shore.

Fotografía: Tak Fujimoto.

Montaje: Craig McKay.

Guión: Ron Nyswaner.

Intérpretes: Tom Hanks, Denzel Washington, Antonio Banderas, Ron Vawter, Robert Ridgely, Joanne Woodward, Jason Robards, Charles Napier, Roberta Maxwell, Buzz Kilman, Karen Finley, Roger Corman, Lisa Summerour...

Color: color.

Duración: 119 minutos.

Género: drama.

Sinopsis: Andrew Beckett, un joven y prometedor abogado de Philadelphia, es despedido del prestigioso bufete en el que trabaja cuando sus jefes se enteran de que ha contraído el sida. Decide entonces demandar a la empresa por despido improcedente, pero en un principio ningún abogado acepta defender su caso.

Productoras: TriStar Pictures / Clinica Estetico Productions.

Enlaces:

https://www.imdb.com/title/tt0107818

https://www.filmaffinity.com/es/film528481.html

\section{Tráiler en inglés}

\section{Introducción}

Aunque esta película ha sido comentada en 2 ocasiones en la Revista de Medicina y Cine; una en $2005^{1}$, Las claves del icono cultural y otra en $2006^{2}$, Visión del SIDA cuando comenzó a ser tratable; en el presente año; 2019, concretamente en marzo, se han cumplido 25 años del estreno de la película Philadelphia (1993) en nuestro país; y afortunadamente muchas cosas han cambiado respecto a la situación que se vivía en esta época. En el presente artículo, pretendemos hacer una reflexión de cómo eran las cosas en 1994 y como lo son ahora 25 años después en 2019.

Dudas sobre las vías de transmisión de la infección y estigmatización social de los pacientes

Una parte muy importante de la película es el reflejo de la realidad social frente a la infección por VIH.

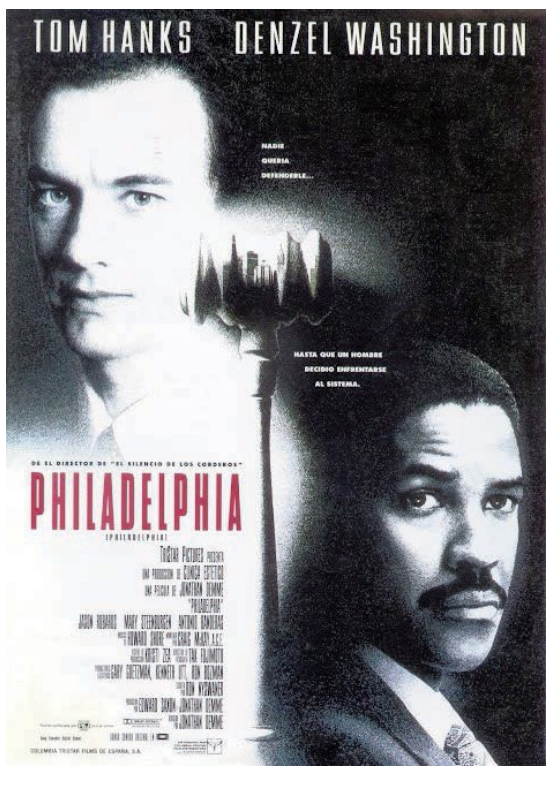

Cartel español.

Por un lado, el temor al contagio y por otro, el rápido conocimiento de las lesiones dérmicas típicas de el Sarcoma de Kaposi.

Aunque en esa época claramente eran conocidas las vías de transmisión de esta infección: sexual, a través de la sangre (transfusiones, hemoderivados, adictos a drogas por vía intravenosa) y vertical (madre-feto), se refleja en la película el miedo al contagio a través de la saliva, del contacto a través del tacto. En la película, tras la visita de Andrew (infectado por VIH) a su abogado; Joseph Miller, 
este se mira la mano (Foto 1) e inmediatamente pide cita con su médico (Foto 2). Esta es la conversación:

- Doctor: El virus VIH solo se transmite a través del intercambio de fluidos corporales: semen, sangre y secreciones vaginales.

- Abogado: Sí, sí pero no es cierto que cada día se están descubriendo cosas nuevas sobre esa enfermedad.
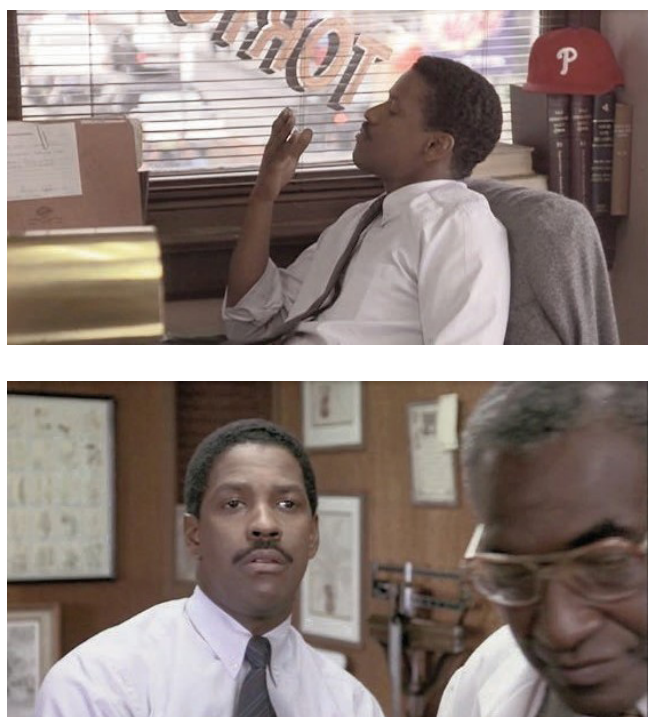

Fotos 1 y 2. Joseph se mira la mano tras haber tocado a Andrew y acude a la consulta de su médico para confirmar que no se ha contagiado.

En nuestro país, en esa época las dudas respecto a las formas de contagio hicieron que muchos de los enfermos fueran considerados como "apestados" y así todo el mundo evitaba cualquier tipo de contacto con ellos. La psicosis del posible contacto determinó que el ministerio de Sanidad y consumo llevara a cabo excelentes campañas de información «Si Da, No Da» (Foto 3) que ya comentó ampliamente nuestro grupo en el artículo publicado en esta misma revista en 2015 sobre la película española $L a$ vida alegre (1987) de Fernando Colomo ${ }^{3}$.

Afortunadamente en el año 2019, la mayor parte de la población no tiene dudas sobre las vías de contagio de esta infección y por otro lado la estigmatización de los pacientes infectados por este virus ha desaparecido. La normalización de la situación hace que los pacientes puedan hacer una vida normal sin ningún tipo de rechazo social ${ }^{4}$.

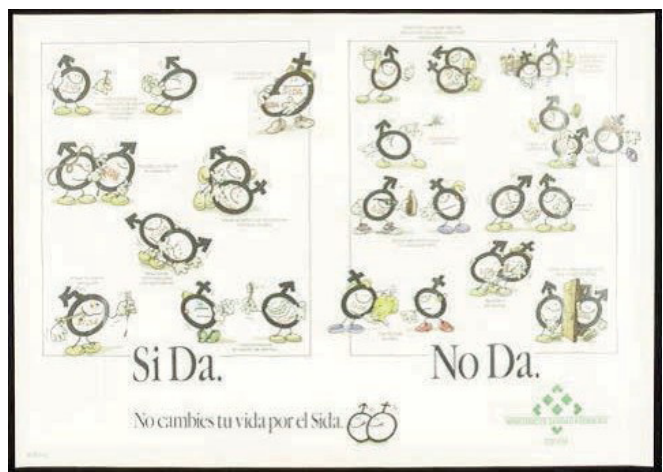

Foto 3. Cartel del Ministerio de Sanidad y Consumo en la campaña publicitaria «Si Da, No Da. Nocambies tu vida por el Sida». 1988.

Manifestaciones clínicas en el momento del diagnóstico e infecciones oportunistas y neoplasias malignas asociadas

En la época que se refleja en la película; primeros años de la década de los noventa, la mayor parte de los pacientes eran diagnosticados de infección VIH-SIDA cuando aparecían signos y síntomas típicos de infecciones oportunistas y de tumores asociados. Las infecciones oportunistas más frecuentes eran la neumonía por Pneumocystis carinii (PCP) (en la actualidad renombrado con Pneumocytis jiroveci), candidiasis esofágica, infecciones por micobacterias (Mycobacterium tuberculosis y $M$. avium-intracelullare (MAI), meningitis por criptococo y toxoplasmosis cerebral. Los tumores malignos más habituales eran el sarcoma de Kaposi ${ }^{5}$; que produjo una importante estigmatización de los pacientes, y los linfomas no Hodgkin.

Afortunadamente en las últimas 2 décadas con la introducción de terapias anti-retrovirales muy efectivas se han conseguido frenar e incluso revertir la inmunodeficiencia producida por este virus y por tanto reducir la incidencia del número de infecciones oportunistas ${ }^{6}$. Así la incidencia de neumonía por PCP y de candidiasis esofágica (las 2 enfermedades oportunistas más frecuentes en este grupo de población) ha pasado en Estados Unidos de 1.000 casos por 100.000 personas y 800 casos por 100.000 respectivamente en los años 2000-2004 a 200 casos en ambas por 100.000 en $2010^{7}$. 
Las terapias antiretrovirales más efectivas han traido consigo un descenso muy importante de los que se llamó canceres definitorios de SIDA; sarcoma de Kaposi ${ }^{5,8,9}$, linfomas no Hodgkin y el cancer de cuello uterino, y no así sino todo lo contrario; un incremento de la incidencia de los canceres no definitorios de SIDA entre los que se hallan el linfoma Hodgkin, el cancer de pulmón así como todos aquellos relacionados con la coinfeccion por VPH (virus del papiloma humano) como cancer anal, vulvar, peneano, oral o faríngeo. En una revisión de cánceres diagnosticados en Estados Unidos en pacientes con SIDA entre 1991 y $2005^{10}$ se ha comprobado una reducción a la mitad de todas la enfermedades malignas desde la aparición de las terapias antiretrovirales de alta eficacia. Así mismo se ha comprobado que de las neoplasias malignas que aparecen ahora la mitad de ellas no son canceres definitorios de SIDA.

\section{Tratamientos farmacológicos y mortalidad}

En la primera década de la aparición de la infección, época que se describe en la película, dentro del arsenal terapéutico solo había aparecido el primer grupo de antiretrovirales (análogos de nucleósicos) y básicamente se utilizaban 3: AZT (zidovudina), DDI (didadosina) y DDC (zalcitabina). Su eficacia no era muy alta debido a la aparición de resistencias con lo que la mortalidad de la infección era muy alta ${ }^{11}$.

En una imagen del filme, se ve a Andrew recibiendo probablemente cotrimoxazol (sulfametoxazol trimetropim) (fármaco utilizado en esa época como profilaxis de la PCP) y al final se ve una imagen del tratamiento diario: AZT (zudovudina; un antiretroviral), Nizoral (ketoconazol; un antifúngico), Megace (megestrol) así como aciclovir y ganciclovir (Foto 4).

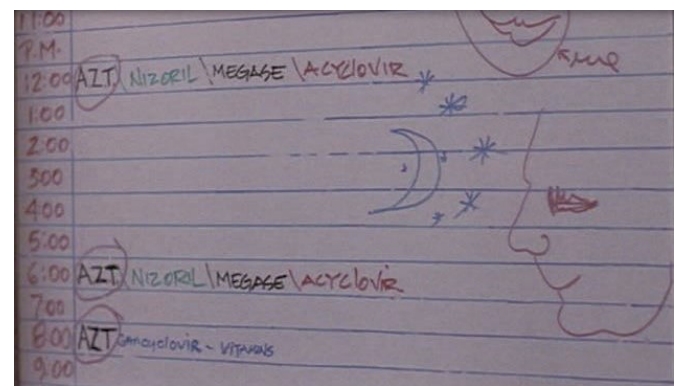

Foto 4. Imagen del tratamiento de Andrew: AZT, Nizoril, Megase, Aciclovir y Ganciglovir.
La zudovudina fue uno de los primeros antiretrovirales, el ketoconazol es un antifungico muy utilizado en esa época para el tratamiento y profilaxis de la candidiasis esofágica, el megestrol es un fármaco estimulador del apetito para intentar combatir los síndromes caquécticos asociados a la infección muy frecuentes en esa época y aciclovir y ganciclovir son antivirales efectivos frente a herpes virus para tratar y prevenir infecciones oportunistas por herpes simplex y citomegalovirus (retinitis).

El cambio sustancial en el tratamiento y la evolución de la infección (mortalidad) por HIV se produjo en 1996 con la aparición del grupo de los inhibidores de proteasas y el establecimiento del denominado tratamiento antiretroviral de alta eficacia conocido en sus siglas en inglés como "HAART»: High Activity Anti Retroviral Therapy ${ }^{12}$. En la actualidad (año 2019) por lo general, el régimen de tratamiento inicial de dicha infección incluye tres o más medicamentos de por lo menos dos clases diferentes ${ }^{13-15}$ :

Inhibidores de la transcriptasa inversa análogos de los nucleósidos (ITIAN)

Inhibidores de la transcriptasa inversa no análogos de los nucleósidos (ITINN)

Inhibidor de la proteasa (IP) Inhibidores de la fusión Antagonistas de CCR5 Inhibidores de la integrasa (INI) Inhibidores posfijación Intensificadores farmacocinéticos

Las pautas actuales más recomendadas por las guías europeas son: 2 ITIAN + INI o 2 ITIAN + ITINN o 2 ITIAN + IP/r. y como pautas alternativas: 2 ITIAN + INI o 2 ITIAN + ITINN o 2 ITIAN + IP/r o IP/ $\mathrm{c}^{13}$.

El pronóstico vital de estos pacientes ha cambiado sustancialmente ${ }^{6}$. En esos años, la expectativa de vida de los pacientes eran pocos años, mientras que actualmente la aparición de múltiples antiretrovirales que son capaces de evitar o enlentecer la progresión de la enfermedad las tasas de mortalidad han disminuido de forma dramática ${ }^{16}$ (Figura 1) hasta tal punto que se puede decir que se ha convertido de una enfermedad mortal a corto plazo a ser una enfermedad crónica ${ }^{12}$.

Por tanto, podríamos decir que en estos 25 años se ha producido un cambio espectacular tanto en las manifestaciones clínicas como en su tratamiento y evolución. Sin embargo, creemos que esta película sigue teniendo la misma vigencia que cuando se realizó en 1993 y es muy útil para que los médicos del siglo XXI conozcan que fue aquella «peste» que diezmó la población en una década: 1985-1995. 
Por otro lado, creemos que la normalización de la enfermedad, ha determinado una cierta relajación por parte de la sociedad en olvidar que la mayor parte de los infectados del siglo XXI será por vía sexual, como así lo demuestran el incremento en los casos y tasas de sífilis y gonococia en los últimos 10 años en nuestro país ${ }^{18}$ (Figura
2). Desde el punto de vista de la salud pública de nuestro país no estaría de más de nuevo campañas publicitarias haciendo referencia al empleo del preservativo como prevención de las enfermedades de transmisión sexual y entre ellas la infección por VIH.

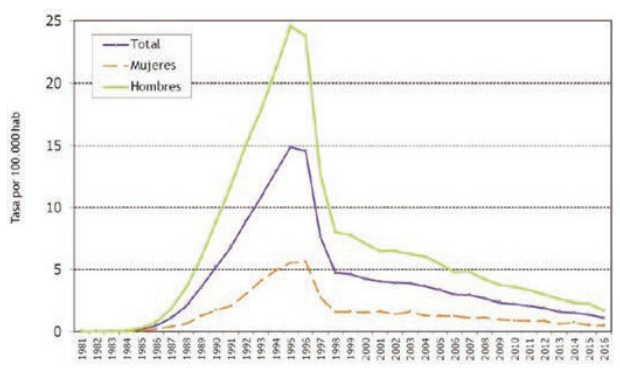

Figura 1. Tasas de mortalidad por HIV/SIDA en España: 1981-2016 tomado del Vigilancia epidemiológica del VIH y Sida en España 2017 del Ministerio de Sanidad y Consumo ${ }^{17}$.
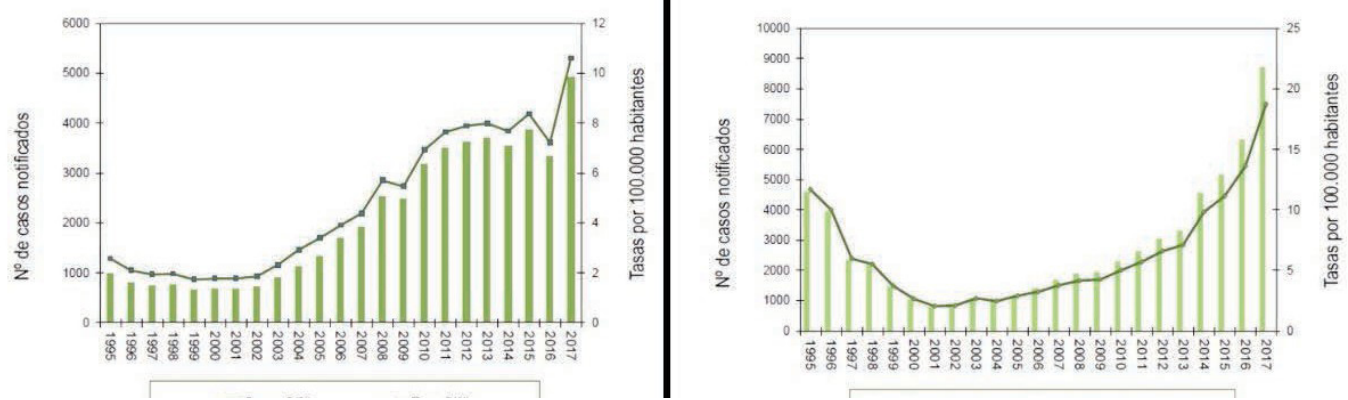

Figura 2. Casos y tasas de sífilis y gonococia en España: 1995-2017. Unidad de vigilancia del VIH y conductas de riesgo. Vigilancia Epidemiológica de las Infecciones de Transmisión Sexual en España, 2018. Centro Nacional. Epidemiología ${ }^{18}$.

\section{Referencias}

1. Aijon Oliva J. Philadelphia (1993): las claves de un icono cultural. Rev Med Cine [internet] 2005;1(4):109-14.

2. Perez Ochoa L. Philadelphia (1993): Visión del SIDA cuando comenzó a ser tratable. Rev Med Cine [internet] 2006;2(1):109-14.

3. Pintor E, Vivas-Rojo E, Herreros B, Gargantilla P. La vida alegre (1987): la importancia del preservativo para prevenir las ETS. Rev Med Cine [internet] 2015; 11(3): 157-62.

4. Fuster-RuizdeApodaca MJ, Molero F, Gil de Montes L, Agirrezabal A, Toledo J, Jaen A, Spanish Group for the Study of
HIV-Related Stigma. Evolution of HIV-related stigma in Spain between 2008 and 2012. AIDS Care. 2014;26(SUPPL. 1):41-5.

5. Holliday AC, Wagner Jr RF. Kaposi's Sarcoma in Film. Rev Med Cine [Internet]. 2013;9(3):107-13.

6. Wolff MJ, Giganti MJ, Cortes CP, Cahn P, Grinsztejn B, Pape JW, et al. A decade of HAART in Latin America: Long term outcomes among the first wave of HIV patients to receive combination therapy. PLoS One. 2017;12(6):e0179769.

7. Masur H. Recurring and emerging questions related to management of HIV-related opportunistic infections. Top Antivir Med. 2018;26(3):79-84. 
8. Seaberg EC, Wiley D, Martínez-Maza O, Chmiel JS, Kingsley $\mathrm{L}$, Tang Y, et al. Cancer incidence in the multicenter aids cohort study before and during the HAART era: 1984 to 2007. Cancer. 2010;116(23):5507-16.

9. Franceschi S, Maso LD, Rickenbach M, Polesel J, Hirschel $B$, Cavassini $M$, et al. Kaposi sarcoma incidence in the Swiss HIV Cohort Study before and after highly active antiretroviral therapy. Br J Cancer. 2008;99(5):800-4.

10. Yarchoan R, Uldrick TS. HIV-Associated Cancers and Related Diseases. N Engl J Med 2018;378(11):1029-41.

11. Greene WC. A history of AIDS: Looking back to see ahead. Eur J Immunol. 2007;37 (1):S94-102.

12. Perez-Hoyos S, Rodríguez-Arenas MÁ, de la Hera MG, Iribarren JA, Moreno S, Viciana P, et al. Progression to AIDS and Death and Response to HAART in Men and Women from a Multicenter Hospital-Based Cohort. J Women's Heal. 2007;16(7):1052-61.

13. European AIDS Clinical Society (EACS). Guía Clínica Versión 9 Octubre de 2017. Eur AIDS Clin Soc. 2017.

14. Johnson SC, MD. Antiretroviral Therapy for HIV Infection: When to Initiate Therapy, Which Regimen to Use, and How to Monitor Patients on Therapy. Top Antivir Med. 2015;23(5):161-7.

15. Lozano F, Domingo P. Tratamiento antirretroviral de la infección por el VIH. Enferm Infecc Microbiol Clin. 2011;29(6):455-65.

16. Área de vigilancia de VIH y conductas de riesgo. Mortalidad por VIH y sida en España, año 2016. Evolución 1981-2016. Madrid: Centro Nacional de Epidemiología/ Subdirección General de Promoción de la Salud y Vigilancia en Salud PúblicaPlan Nacional sobre el Sida; 2018.

17. Área de Vigilancia de VIH y Comportamientos de Riesgo. Vigilancia Epidemiológica del VIH y sida en España 2017: Sistema de Información sobre Nuevos Diagnósticos de VIH y Registro Nacional de Casos de Sida. Madrid: Plan Nacional sobre el Sida - D.G. de Salud Pública, Calidad e Innovación / Centro Nacional de Epidemiología - ISCIII.2018.

18. Unidad de vigilancia del VIH y conductas de riesgo. Vigilancia epidemiológica de las infecciones de transmisión sexual, 2016. Madrid: Centro Nacional de Epidemiología/Subdirección General de Promoción de la Salud y Vigilancia en Salud Pública - Plan Nacional sobre el Sida; 2018.

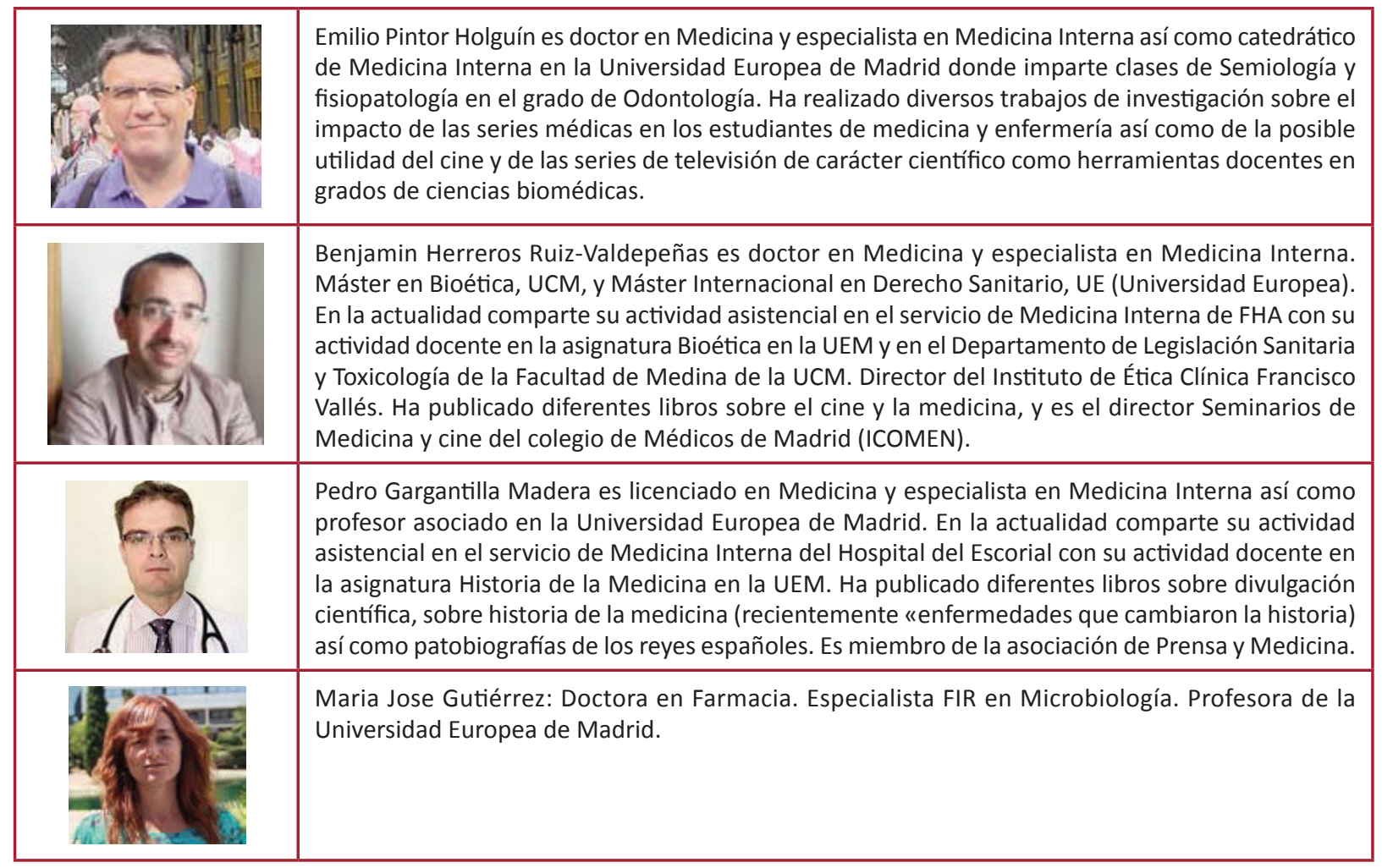

